\title{
Osteoporosetherapie
}

\section{Stabile Knochen bis ins hohe Alter}

- Die medikamentöse Osteoporosetherapie kann viele Frakturen verhindern. Leider ist ihr aber oft eine schlechte Compliance beschieden. Neue Substanzen wie Denosumab haben das Potenzial für mehr Akzeptanz und eine verbesserte Wirksamkeit.

„Wir schaffen es, mit der Osteoporosetherapie im Jahr 2012 die Zahl der Wirbelkörperfrakturen um 50-70\%, der Schenkelhalsbrüche um $40 \%$ und der Brüche insgesamt um 20\% zu reduzieren", erklärte Prof. Johannes Pfeilschifter, Essen. Damit konnte auch die Sterblichkeit um 30\% verringert und die Lebensqualität verbessert werden. Pfeilschifter kritisierte allerdings die in Deutschland deutlich schlechtere Versorgung alter Hochrisikopatienten - im Vergleich zu anderen Ländern.

\section{Therapeutische Optionen}

Prof. Lorenz Hofbauer, Dresden, wies darauf hin, dass es verschiedene Optionen gibt, in den Knochenstoffwechsel einzugreifen: bei der Knochenneubildung oder der Knochenresorption. Dazu gehören z. B. orale Bisphosphonate. Deren Problem ist

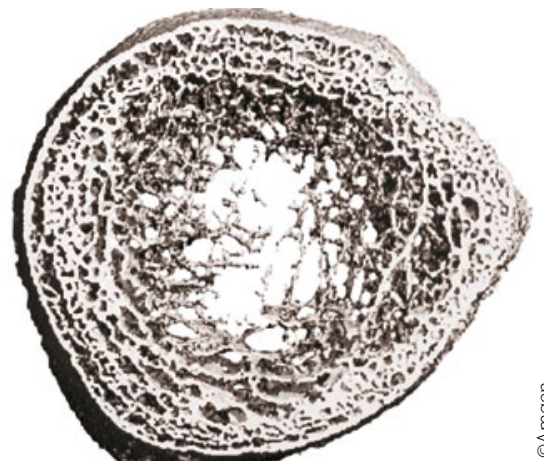

Osteoporotischer Knochen.

allerdings die geringe Langzeitcompliance und der gewöhnungsbedürftige Einnahmemodus. Neue Therapieformen sind nicht nur effektiver, sondern auch komfortabler in der Anwendung, erklärte Hofbauer. Unter den Antiresorptiva gibt es z. B. den Cathepsin-K-Inhibitor Odanacatib, der sich in der Phase-III-Erprobung befindet, oder den bereits zugelassenen RANKLigand-Inhibitor Denosumab (Prolia®).

Denosumab wird in der Dosierung 60 mg alle sechs Monate subkutan injiziert. Grundlage für die Zulassung war die placebokontrollierte Phase-III-Studie FREEDOM
(N Engl J Med 2009; 361:756-65). Nach drei Jahren hatten sich neue Wirbelkörperfrakturen relativ um $68 \%$, nicht vertebrale Frakturen um 20\% und Hüftfrakturen um $40 \%$ seltener ereignet. Die FREEDOM-Studie (J Bone miner Res. 2011 Nov 23. Epub ahead of print) wurde über die drei Jahre hinaus für zwei Jahre offen fortgeführt. Auch dabei bestätigte sich die niedrige Inzidenz vertebraler und nicht vertrebraler Frakturen sowie die gute Verträglichkeit.

Die DECIDE-Studie (Journal of Bone and Mineral Research 2010; 25(1): 72-81) untersuchte Veränderungen von Knochendichte und -umsatz bei postmenopausalen Patientinnen. Nach Vorbehandlung mit Alendronat waren sie mit Denosumab bzw. Alendronat weiterbehandelt worden. „In jedem gemessenen Parameter von Hüfte, Wirbelsäule, Schenkelhals und distalem Radius stand die Denosumab-Gruppe nach einem Jahr besser da," sagte Hofbauer.

- Martin Bischoff

Quelle: Symposium „Osteoporose - eine komplexe Erkrankung auf dem Vormarsch", DGIMKongress, Wiesbaden, April 2012 (Veranstalter: Amgen und GlaxoSmithKline)

\section{Online-Fortbildung}

\section{Wiederkehrende ischämische Beschwerden}

\begin{abstract}
- Haben Patienten Beschwerden einer Mangeldurchblutung im Herzen, kommen aber vom Kardiologen ohne interventionspflichtigen Befund wieder, stellt sich die Frage, was tun? „Da ist keine Stenose", sagt der Kardiologe, „ich habe Beschwerden", sagt der Patient.
\end{abstract}

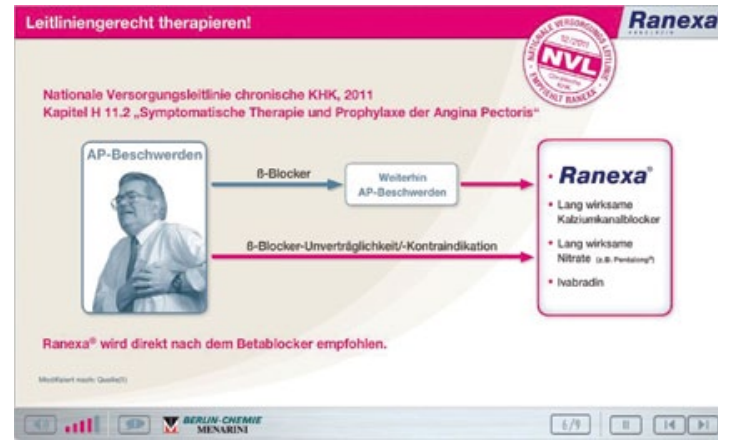
Was steckt hinter einer wiederkehrenden kardialen Ischämie, was ist der Pathomechanismus, welche therapeutischen Optionen gibt es, welche antiischämischen Wirkstoffe sind verfügbar, wie wirkt Ranolazin? Antwor- ten bietet in rund zehn Minuten die Online-Fortbildung „kardiale Ischämie“ von Berlin-Chemie unter: www.ranexa-schulung.de, Zugangscode: ranolazin.

\section{Kurz notiert}

Nasaler Grippeimpfstoff für 2012/2013 - Der nasale InfluenzaImpfstoff Fluenz $z^{\circledast}$ hat von der Europäischen Arzneimittelbehörde EMA die saisonale Zulassung für die Grippesaison 2012/2013 erhalten. Damit kann in Kürze erstmals ein InfluenzaImpfstoff für Kinder und Jugendliche ab einem Lebensalter von 24 Monaten bis zum vollendeten 18. Lebensjahr, der nasal appliziert wird, in den deutschen Markt eingeführt werden. Der Berufsverband der Kinder- und Jugendärzte (BVKJ) spricht sich dafür aus, dass die nasale Impfung wegen seiner besseren Wirksamkeit auch gesetzlich versicherten Kindern von den Krankenkassen zur Verfügung gestellt werden sollte. 